\title{
Flood Frequency Analysis of Upper Krishna River Basin catchment area using Log Pearson Type III Distribution
}

\author{
B. K. Sathe ${ }^{1}$ M. V. Khire ${ }^{2}$ R. N. Sankhua ${ }^{3}$ \\ ${ }^{I}$ Research Scholar, CSRE, IIT-Bombay ${ }^{I}$ Corresponding author \\ ${ }^{2}$ Associate Professor, CSRE, IIT-Bombay, \\ ${ }^{3}$ Director, National Water Academy, Pune-411024
}

\begin{abstract}
In this study, a flood frequency analysis of Upper Krishna River basin in India is carried out by LogPearson Type-III probability distribution method. This method is a statistical technique for fitting frequency distribution data to predict the flood for a river at some site. In Upper Krishna River The annual peak flood series data for 10 years varying over period 1965 to 2010 for 7 important stations such as Karad,Warna, Arjunwad, Kurundwad, Warungi, Terwad, Sadagli are analysed.out of these seven stations Arjunwad and Kurundwad river gauging stations are important for flash flood point of view. The probability distribution function was applied to return periods $(T)$ of $T=2$ yrs, $5 y r s, 10 y r s, 25 y r s, 50 y r s, 100 y r s$ and 200 yrs commonly used in for engineering design of hydraulic structures. These values are useful for hydraulic design of structures in the catchment area and for storm water management. The model relates the expected discharge to return period for all tributaries of Upper Krishna River basin.
\end{abstract}

Keywords: - design discharge, flood frequency, gauge discharge, Log Person Type III, probability

\section{INTRODUCTION}

Floods are the most common natural disasters that affect societies around the world. Dilley et al. (2005) estimated that more than one-third of the world's land area is flood prone affecting some 82 percent of the world's population. About 196 million people in more than 90 countries are exposed to catastrophic flooding, and that some 170,000 deaths were associated with floods worldwide between 1980 and 2000 UNDP (2004). These figures show that flooding is a major concern in many regions of the world. To protect lives and properties it is needful for hydraulic structures to be constructed to safely handle an approximate percentage of the probable maximum flood.. As much of the hydraulic data like flow rate (discharge) and rainfall are statistical in nature, statistical methods are most frequently needed to be used often with the goal of fitting a statistical distribution to the data [11]. Design flood is the discharge adopted for the design of a hydraulic structure and it is obviously very costly to design any hydraulic structure so as to make it safe against the maximum flood possible in the catchment. [3]

The procedure for estimating the frequency of occurrence (return period) of a hydrological event such as flood is known as (flood) frequency analysis. Though the nature of most hydrological events (such as rainfall) is erratic and varies with time and space, it is commonly possible to predict return periods using various probability distributions [17]. Flood frequency analysis was developed as a statistical tool to help engineers, hydrologists, and watershed managers to deal with this uncertainty. Flood frequency is utilized to determine how often a storm of a given magnitude would occur. It is an important tool for the building and design of the safest possible structures (e.g. dams, bridges, culverts, drainage systems etc.) because the design of such structures demands knowledge of the likely floods which the structure would have to withstand during its estimated economic useful life[6].

In particular, analysis of annual one day maximum rainfall and consecutive maximum days rainfall of different return periods ( typically 2 to 100 years) is a basic tool for safe and economic planning and design of small dams, bridges, culverts, irrigation and drainage work as well as for determining drainage coefficients [4]. In this study the log Pearson Type III probability distribution function have been used to model the annual peak discharge data of Upper Krishna River Basin. The main objective of the study was to perform flood frequency analysis of the river catchment using annual peak flow or maximum discharge data obtained in the river in the water years 1965 to 2010. The specific objectives of the study were:

(i) Fit the Log Pearson Type III probability distribution to the annual peak discharge data and hence

(ii) Predict design for the following return periods ( $\mathrm{T}=2 \mathrm{yrs}, 5 \mathrm{yrs}, 10 \mathrm{yrs}, 25 \mathrm{yrs}, 50 \mathrm{yrs}, 100 \mathrm{yrs}$ and 200 years)

\section{STUDY AREA}

The study area comprises of an upland watershed and a major tributary of Krishna River in the upper Krishna basin. The river has its source in the Western Ghats on the leeward side of the mountains Maharashtra, 
India. The river is $310 \mathrm{kms}$ long and the catchment covers an area of 14,539 sq. km falling in Survey of India (SOI) toposheet No: $47 / \mathrm{K}, 47 / \mathrm{L}, 47$ / P on 1:250,000 scale. The investigated area is enclosed between latitudes $17^{\circ} 18^{\prime} \mathrm{N}$ and $16^{\circ} 15^{\prime} \mathrm{N}$ and longitudes $73^{\circ} 50^{\prime} \mathrm{E}$ and $75^{\circ} 54^{\prime} \mathrm{E}$. (Figure 2)

The annual peak flood series data for 10 years varying over period 1965 to 2010 for 7 important stations such as Karad, Warna, Arjunwad, Kurundwad, Warungi of Upper Krishna basin. The data were collected from the Maharashtra state irrigation department

\section{HEORY OF LOG-PEARSON TYPE III PROBABILITY DISTRIBUTION}

The Log-Pearson Type III distribution is a statistical technique for fitting frequency distribution data to predict the design flood for a river at some site. Once the statistical information is calculated for the river site, a frequency distribution can be constructed. The probabilities of floods of various sizes can be extracted from the curve. The advantage of this particular technique is that extrapolation can be made of the values for events with return periods well beyond the observed flood events. This technique is the standard technique used by Federal Agencies in the United States.

\section{FloOd Discharge COMPUTATIONAL ANALYSIS}

The Log-Pearson Type III distribution is calculated using the general equation

$$
\mathbf{X}=\bar{X}+\mathbf{K} \boldsymbol{\sigma} \quad \text { ( } 1 \text { ) }
$$

Where $\mathrm{k}=$ frequency factor determined from Tables No5. The model parameters $\overline{\boldsymbol{X}}$, standard deviation and the skew coefficient $(\mathrm{g})$ are computed from $\mathrm{n}$ observations $\mathrm{X}$, with the following formula

$$
\begin{aligned}
& \bar{X}=\frac{1}{n} \sum_{i=1}^{n} X i \\
& \sigma=\left\lfloor\frac{1}{(n-1)} \sum(X-\bar{X})^{2}\right]^{1 / 2} \\
& \mathbf{g}=\frac{\frac{1}{(n-1)} \sum(X-\bar{X})^{2}}{(n-1)(n-2) \sigma^{2}}
\end{aligned}
$$

However, the Log Pearson Type III distribution of $\mathrm{X}$ which has been widely adopted to reduce skewness is equivalent to applying Pearson Type III to the transformed variable $\log \mathrm{X}$ and it is represented in the literature

(e.g. HannC.T.(1977) Das and Saikia (2009); Jagadesh and Jayaram (2009); Wurbs and James, 2009) as:

$\log X=\overline{\log X}+\mathrm{K} \sigma_{\log X}$

(5)

where $\mathrm{X}$ is the flood discharge value of some specified probability, $\overline{\log X}$ is the average of the $\log \mathrm{X}$ discharge values, $\mathrm{K}$ is frequency factor. $\boldsymbol{\sigma}_{\log \mathrm{X}}$ is the standard deviation of $\log \mathrm{x}$ values. The frequency factor $\mathrm{K}$ is a function of skewness coefficient and return period and can be read from published tables (Table 5) developed by integrating the appropriate probability density function. The flood magnitude for various return periods are found by solving the general equation. The mean,standard deviation of the data and skewness coefficient can be calculated using the following

formula

$$
\begin{aligned}
& \overline{\log X}=\frac{\sum \log X}{n} \\
& \varphi_{\log X}=\left[\frac{\sum(\log X i-\log X)^{2}}{(n-1)}\right]^{1 / 2} \\
& g=\frac{\sum(\log X i-\overline{\log X})^{2}}{(n-1)(n-2) \sigma_{\log x}^{3}}
\end{aligned}
$$

Where $\mathrm{n}$ is the number of entries of $\mathrm{X}$ the flood of some specified probability $\log \mathrm{X} \mathrm{i}$ is the average of the $\log \mathrm{x}$ discharge value

\section{METHODOLOGY}

Log Pearson Type III distribution and given in equation

$$
T=\frac{n+0.2}{m-0.4}
$$

where $\mathrm{n}$ is the number of years of record and $\mathrm{m}$ is the rank obtained by arranging the annual flood series in descending order of magnitude with the maximum being assigned the rank of 1.

In carrying out the flood frequency analysis using the log-Pearson Type III distribution, the following steps suggested by Jagadesh and Jayaram (2009) were adopted: 
Flood Frequency Analysis of Upper Krishna River Basin catchment area using Log Pearson Type III

(i) The annual flood series (Xii) were assembled

(ii) The logarithms of the annual flood series were calculated as yi $=\log \mathrm{Xi}$

(iii) The mean $y$, the standard deviation $y$ and skew coefficient Cs of the logarithm yi were calculated.

(iv) The logarithms of the flood discharge i.e. log Qi for each of the several chosen probability level $P_{j}$ were calculated

using the following frequency formula

$\log _{\mathrm{i}} Q \square \square \mathrm{y} \square \square \mathrm{j}$ Khhere $\mathrm{K}_{\mathrm{j}}$ is the frequency factor, a function of the probability Pj and Skewness coefficient Table 5 shows the frequency factor $(\mathrm{k})$ for ten selected probability levels in the range from 0.5 to $95 \%$ and skewness coefficient in the range from -3 . To 3.0

(v) The flood discharge $Q_{j}$ for each Pi probability level (return period $T_{j}$ ) is obtained by taking antilogarithms of the $\log \varphi$ values.

Table 1: Annual peak Discharge data for river gauging stations $\left(\mathrm{m}^{3} / \mathrm{s}\right)$

\begin{tabular}{|c|c|c|c|c|c|c|c|c|}
\hline \multirow[t]{2}{*}{ Sr.No. } & \multirow{2}{*}{$\begin{array}{l}\text { Water } \\
\text { Year }\end{array}$} & \multicolumn{7}{|c|}{ River gauging Stations } \\
\hline & & $\begin{array}{l}\text { Arjunwad } \\
\text { (krishna) }\end{array}$ & $\begin{array}{l}\text { Karad } \\
\text { (Krishna) }\end{array}$ & $\begin{array}{l}\text { Kurundwad } \\
\text { (Krishna) }\end{array}$ & $\begin{array}{l}\text { Samdoli } \\
\text { (Warna) }\end{array}$ & $\begin{array}{l}\text { Sadagli } \\
\text { (Dudh } \\
\text { ganga) }\end{array}$ & $\begin{array}{l}\text { Terwad } \\
\text { (Panchganga) }\end{array}$ & $\begin{array}{l}\text { Warunji } \\
\text { (Koyna) }\end{array}$ \\
\hline 1 & $\begin{array}{l}1965- \\
1966 \\
\end{array}$ & \multirow{4}{*}{$\begin{array}{l}\text { Data not } \\
\text { Available }\end{array}$} & 4760 & \multirow{5}{*}{$\begin{array}{l}\text { Data not } \\
\text { available }\end{array}$} & & \multirow{4}{*}{$\begin{array}{l}\text { Data not } \\
\text { Available }\end{array}$} & \multirow{15}{*}{$\begin{array}{l}\text { Data not } \\
\text { Available }\end{array}$} & \multirow[t]{2}{*}{$\begin{array}{l}\text { Data not } \\
\text { Available }\end{array}$} \\
\hline 2 & $\begin{array}{c}1966- \\
1967\end{array}$ & & 2673 & & & & & \\
\hline 3 & $\begin{array}{l}1967- \\
1968\end{array}$ & & 4482 & & 2000 & & & 3830 \\
\hline 4 & $\begin{array}{c}1968- \\
1969\end{array}$ & & 1527 & & 1068 & & & 757.4 \\
\hline 5 & $\begin{array}{l}1969- \\
1970\end{array}$ & 3850 & 4267 & & 1650 & 1225 & & 1605 \\
\hline 6 & $\begin{array}{c}1970- \\
1971 \\
\end{array}$ & 5079 & 2428 & & 1555 & 1320 & & 1492 \\
\hline 7 & $\begin{array}{l}1971- \\
1972\end{array}$ & 2936 & 2156 & & 1220 & 1190 & & 1386 \\
\hline 8 & $\begin{array}{l}1972- \\
1973\end{array}$ & 3989 & 2190 & 4239 & 1219 & 1230 & & 1523 \\
\hline 9 & $\begin{array}{l}1973- \\
1974\end{array}$ & 4950 & 3051 & 5659 & 2250 & 1397 & & 1900 \\
\hline 10 & $\begin{array}{l}1974- \\
1975 \\
\end{array}$ & 2469 & 3101 & 3436 & 1528 & 1304 & & 1583 \\
\hline 11 & $\begin{array}{l}1975- \\
1976\end{array}$ & 5270 & 3231 & 5781 & 2050 & 1759 & & 1681 \\
\hline 12 & $\begin{array}{l}1976- \\
1977\end{array}$ & 4890 & 4562 & 6854 & 2007 & 1103 & & 2780 \\
\hline 13 & $\begin{array}{l}1977- \\
1978\end{array}$ & 3460 & 3422 & 4953 & 1548 & 1129 & & 1610 \\
\hline 14 & $\begin{array}{l}1978- \\
1979 \\
\end{array}$ & 2579 & 2187 & 3584 & 1418 & 1244 & & 1576 \\
\hline 15 & $\begin{array}{c}1979- \\
1980\end{array}$ & 4904 & 2435 & 6070 & 1436 & 1429 & & 1317 \\
\hline 16 & $\begin{array}{l}1980- \\
1981 \\
\end{array}$ & 3800 & 2848 & 5175 & 1747 & 1103 & 1918 & 1868 \\
\hline 17 & $\begin{array}{l}1981- \\
1982\end{array}$ & 3362 & 1942 & 5166 & 1741 & 1457 & 1790 & 1262 \\
\hline 18 & $\begin{array}{l}1982- \\
1983\end{array}$ & 2241 & 1261 & 3438 & 1472 & 1368 & 1431 & 1156 \\
\hline 19 & $\begin{array}{l}1983- \\
1984\end{array}$ & 3345 & 1931 & 5111 & 1682 & 2435 & 1545 & 1493 \\
\hline 20 & $\begin{array}{c}1984- \\
1985\end{array}$ & 3284 & 1797 & 4427 & 1715 & 1514 & 1795 & 1334 \\
\hline
\end{tabular}


Flood Frequency Analysis of Upper Krishna River Basin catchment area using Log Pearson Type III

\begin{tabular}{|c|c|c|c|c|c|c|c|c|}
\hline 21 & $\begin{array}{c}1985- \\
1986\end{array}$ & 3085 & 1363 & 4414 & 1530 & 1421 & 1570 & 1054 \\
\hline 22 & $\begin{array}{l}1986- \\
1987\end{array}$ & 2868 & 1801 & 4587 & 1347 & 1048 & 2050 & 1284 \\
\hline 23 & $\begin{array}{l}1987- \\
1988\end{array}$ & 2351 & 1323 & 3290 & 1080 & 872.6 & 1392 & 1073 \\
\hline 24 & $\begin{array}{c}1988- \\
1989\end{array}$ & 4997 & 3205 & 4870 & 2025 & 1810 & 2080 & 2590 \\
\hline 25 & $\begin{array}{c}1989- \\
1990\end{array}$ & 4954 & 2504 & 6000 & 2412 & 2100 & 2205 & 1600 \\
\hline 26 & $\begin{array}{c}1990- \\
1991\end{array}$ & 6500 & 4361 & 5760 & 1675 & 1452 & 2250 & 2530 \\
\hline 27 & $\begin{array}{c}1991- \\
1992\end{array}$ & 5938 & 3150 & 6322 & 2081 & 1379 & 2553 & 1800 \\
\hline 28 & $\begin{array}{c}1992- \\
1993\end{array}$ & 2928 & 2127 & 3919 & 1178 & 773.1 & 1306 & 1238 \\
\hline 29 & $\begin{array}{c}1993- \\
1994\end{array}$ & 2843 & 1812 & 4051 & 1463 & 1058 & 1447 & 1023 \\
\hline 30 & $\begin{array}{l}1994- \\
1995\end{array}$ & 6300 & 3915 & 5730 & 2235 & 1900 & 2680 & 2675 \\
\hline 31 & $\begin{array}{c}1995- \\
1996\end{array}$ & 2550 & 1356 & 2796 & 869.0 & 710.9 & 1170 & 772.1 \\
\hline 32 & $\begin{array}{l}1996- \\
1997\end{array}$ & 3560 & 2944 & 5000 & 1610 & 1180 & 1900 & 1566 \\
\hline 33 & $\begin{array}{c}1997- \\
1998\end{array}$ & 4780 & 5954 & 6800 & 1710 & 1350 & 3590 & 3529 \\
\hline 34 & $\begin{array}{c}1998- \\
1999\end{array}$ & 2411 & 1541 & 3000 & 897.5 & 685.5 & 1051 & 828 \\
\hline 35 & $\begin{array}{l}1999- \\
2000\end{array}$ & 3193 & 2111 & 3725 & 1199 & 1110 & 1540 & 1397 \\
\hline 36 & $\begin{array}{c}2000- \\
2001\end{array}$ & 1747 & 774.1 & 2852 & 1001 & 853.6 & 890.0 & 676.7 \\
\hline 37 & $\begin{array}{l}2001- \\
2002\end{array}$ & 1764 & 911.2 & 2594 & 637.2 & 594.6 & 1150 & 623.3 \\
\hline 38 & $\begin{array}{l}2002- \\
2003\end{array}$ & 1678 & 1121 & 3014 & 694.6 & 827.9 & 1443 & 830.2 \\
\hline 39 & $\begin{array}{c}2003- \\
2004\end{array}$ & 1333 & 936.6 & 2275 & 772.1 & 537.7 & 655.0 & 626.7 \\
\hline 40 & $\begin{array}{l}2004- \\
2005\end{array}$ & 4211 & 4163 & 4650 & 1261 & 1287 & 1832 & 2716 \\
\hline 41 & $\begin{array}{l}2005- \\
2006\end{array}$ & 9381 & 6312 & 10092 & 3064 & 2200 & 3340 & 4641 \\
\hline 42 & $\begin{array}{l}2006- \\
2007 \\
\end{array}$ & 7505 & 6708 & 8819 & 2010 & 1978 & 2797 & 4973 \\
\hline 43 & $\begin{array}{c}2007- \\
2008\end{array}$ & 3943 & 3868 & 5673 & 1569 & 891.2 & 1821 & 2243 \\
\hline 44 & $\begin{array}{c}2008- \\
2009\end{array}$ & 3357 & 2884 & 5743 & 1403 & 1013 & 1952 & 1887 \\
\hline
\end{tabular}

Table 2 Computation of statistical parameters for Warunji (Koyna)

\begin{tabular}{|c|l|c|r|r|r|r|r|}
\hline $\begin{array}{l}\text { Rank } \\
(\mathbf{m})\end{array}$ & $\begin{array}{l}\text { Water } \\
\text { Year }\end{array}$ & $\begin{array}{l}\text { Qmax } \\
(\mathbf{X ~ m 3 / s})\end{array}$ & $\begin{array}{l}\mathbf{y}=\mathbf{l o g} \\
\mathbf{X}\end{array}$ & \multicolumn{1}{|c|}{$(\boldsymbol{y}-\overline{\mathbf{y}}) \mathbf{2}$} & $(\boldsymbol{y}-\overline{\mathbf{y}}) \mathbf{3}$ & $\mathbf{T}=\frac{\boldsymbol{n + 0 . 2}}{\boldsymbol{m - 0 . 4}}$ & \multicolumn{1}{|c|}{$\mathbf{\mathbf { 1 0 0 }}$} \\
\hline $\mathbf{1}$ & $2006-2007$ & 4973 & 3.6966 & 0.2562 & 0.1296 & 70.33 & 1.42 \\
\hline $\mathbf{2}$ & $2005-2006$ & 4641 & 3.6666 & 0.226 & 0.1079 & 26.37 & 3.79 \\
\hline $\mathbf{3}$ & $1967-1968$ & 3830 & 3.5831 & 0.1542 & 0.0605 & 16.23 & 6.16 \\
\hline
\end{tabular}


Flood Frequency Analysis of Upper Krishna River Basin catchment area using Log Pearson Type III

\begin{tabular}{|c|c|c|c|c|c|c|c|}
\hline 4 & 1997-1998 & 3529 & 3.5476 & 0.1275 & 0.0455 & 11.72 & 8.53 \\
\hline 5 & 1976-1977 & 2780 & 3.4440 & 0.0643 & 0.0163 & 9.173 & 10.90 \\
\hline 6 & 2004-2005 & 2716 & 3.4339 & 0.0592 & 0.0144 & 7.535 & 13.27 \\
\hline 7 & 1994-1995 & 2675 & 3.4273 & 0.0561 & 0.0132 & 6.39 & 15.63 \\
\hline 8 & 1988-1989 & 2590 & 3.4132 & 0.0496 & 0.0110 & 5.55 & 18.00 \\
\hline 9 & 1990-1991 & 2530 & 3.4031 & 0.0452 & 0.0096 & 4.90 & 20.37 \\
\hline 10 & 2007-2008 & 2243 & 3.3508 & 0.0257 & 0.0041 & 4.39 & 22.74 \\
\hline 11 & 1973-1974 & 1900 & 3.2787 & 0.0077 & 0.00068 & 3.98 & 25.11 \\
\hline 12 & 2008-2009 & 1887 & 3.2757 & 0.0072 & 0.0006 & 3.63 & 27.48 \\
\hline 13 & 1980-1981 & 1868 & 3.2713 & 0.0065 & -0.0018 & 3.34 & 29.85 \\
\hline 14 & 1991-1992 & 1800 & 3.2552 & 0.004 & 0.0002 & 3.10 & 32.22 \\
\hline 15 & 1975-1976 & 1681 & 3.2255 & 0.0012 & $4.33148 \mathrm{E}-05$ & 2.89 & 34.59 \\
\hline 16 & $1977-1978$ & 1610 & 3.2068 & 0.0002 & $4.39277 \mathrm{E}-06$ & 2.70 & 36.96 \\
\hline 17 & 1969-1970 & 1605 & 3.2054 & 0.0002 & $3.39299 \mathrm{E}-06$ & 2.54 & 39.33 \\
\hline 18 & 1989-1990 & 1600 & 3.2041 & 0.0001 & $2.55537 \mathrm{E}-06$ & 2.39 & 41.70 \\
\hline 19 & 1974-1975 & 1583 & 3.1994 & $8.15857 \mathrm{E}-0$ & $7.36921 \mathrm{E}-07$ & 2.26 & 44.07 \\
\hline 20 & 1978-1979 & 1576 & 3.1975 & $5.05205 \mathrm{E}-05$ & $3.59089 \mathrm{E}-07$ & 2.15 & 46.44 \\
\hline 21 & 1996-1997 & 1566 & 3.1947 & $1.88645 \mathrm{E}-05$ & $8.19344 \mathrm{E}-08$ & 2.048 & 48.81 \\
\hline 22 & $1972-1973$ & 1523 & 3.1826 & $6.00397 \mathrm{E}-05$ & $-4.6522 \mathrm{E}-07$ & 1.95 & 51.18 \\
\hline 23 & 1983-1984 & 1493 & 3.1740 & 0.00026 & $\begin{array}{r}-4.40177 \mathrm{E}- \\
06 \\
\end{array}$ & 1.86 & 53.55 \\
\hline 24 & 1970-1971 & 1492 & 3.1737 & 0.00027 & $\begin{array}{r}-4.64043 \mathrm{E}- \\
06 \\
\end{array}$ & 1.78 & 55.92 \\
\hline 25 & 1999-2000 & 1397 & 3.1451 & 0.00204 & $\begin{array}{r}-9.26647 \mathrm{E}- \\
05 \\
\end{array}$ & 1.71 & 58.29 \\
\hline 26 & 1971-1972 & 1386 & 3.1417 & 0.00237 & -0.00011 & 1.64 & 60.66 \\
\hline 27 & 1984-1985 & 1334 & 3.1251 & 0.00426 & -0.0002 & 1.58 & 63.033 \\
\hline 28 & 1979-1980 & 1317 & 3.1195 & 0.00502 & -0.00035 & 1.52 & 65.40 \\
\hline 29 & 1986-1987 & 1284 & 3.1085 & 0.00670 & -0.0005 & 1.47 & 67.77 \\
\hline 30 & 1981-1982 & 1262 & 3.1010 & 0.00799 & -0.00071 & 1.42 & 70.14 \\
\hline 31 & 1992-1993 & 1238 & 3.0927 & 0.00955 & -0.00093 & 1.37 & 72.51 \\
\hline 32 & 1982-1983 & 1156 & 3.0629 & 0.01625 & -0.00207 & 1.33 & 74.88 \\
\hline 33 & 1987-1988 & 1073 & 3.0305 & 0.02555 & -0.00408 & 1.29 & 77.25 \\
\hline 34 & 1985-1986 & 1054 & 3.0228 & 0.02809 & -0.00470 & 1.25 & 79.62 \\
\hline 35 & 1993-1994 & 1023 & 3.0098 & 0.03260 & -0.00588 & 1.21 & 81.99 \\
\hline 36 & 2002-2003 & 830.2 & 2.9191 & 0.07358 & -0.01996 & 1.185 & 84.36 \\
\hline 37 & 1998-1999 & 828 & 2.9180 & 0.0742 & -0.02021 & 1.153 & 86.72 \\
\hline 38 & 1995-1996 & 772.1 & 2.8876 & 0.09167 & -0.0277 & 1.12 & 89.09 \\
\hline 39 & 1968-1969 & 757.4 & 2.8793 & 0.09679 & -0.03011 & 1.093 & 91.46 \\
\hline 40 & 2000-2001 & 676.7 & 2.8303 & 0.12963 & -0.04667 & 1.0656 & 93.83 \\
\hline 41 & 2003-2004 & 626.7 & 2.7970 & 0.15475 & -0.0608 & 1.039 & 96.20 \\
\hline 42 & 2001-2002 & 623.3 & 2.7946 & 0.15661 & -0.0619 & 1.014 & 98.57 \\
\hline & Average & $\begin{array}{l}\bar{X}=1769.7 \\
48\end{array}$ & $\begin{array}{r}\bar{y}=3.1 \\
904 \\
\end{array}$ & $\begin{array}{l}\text { Sum }= \\
2.011\end{array}$ & $\begin{array}{l}\text { Sum = } \\
0.1250\end{array}$ & & \\
\hline & & \multicolumn{2}{|c|}{$\begin{array}{l}\text { Std. deviation } \\
\sigma=0.2214 \\
\end{array}$} & \multicolumn{2}{|c|}{ Skew coeff. $g=1.790$} & & \\
\hline
\end{tabular}


Flood Frequency Analysis of Upper Krishna River Basin catchment area using Log Pearson Type III

Table 3: Sample Calculation of Discharges for return periods for Warunji (Koyna)

\begin{tabular}{|c|c|c|c|c|c|c|}
\hline $\begin{array}{l}\text { Gauge } \\
\text { Station }\end{array}$ & $\begin{array}{l}\text { Return } \\
\text { period } \\
\text { T(yrs) }\end{array}$ & $\begin{array}{l}\text { Probability } \\
\text { P }(\%)\end{array}$ & $\begin{array}{l}\text { Frequency factor } \\
\mathrm{K} \\
\mathrm{g}=\mathbf{1 . 7 9 0}\end{array}$ & $\begin{array}{l}\quad \text { yi }=\log \mathrm{Q} \\
\text { yi }=\overline{\boldsymbol{y}}+ \\
\boldsymbol{K} X \boldsymbol{S y}\end{array}$ & $\begin{array}{c}\mathbf{X i}=\mathbf{Q} \\
\mathrm{m} 3 / \mathrm{s}\end{array}$ & $\begin{array}{l}\text { Relation between } \\
\text { Expected Discharge } \\
\text { and Return Period }\end{array}$ \\
\hline \multirow{7}{*}{ Warungi } & 2 & 50 & -0.274 & 3.129 & 1345.860 & \multirow{7}{*}{$y=1980 . \ln (x)-1392$} \\
\hline & 5 & 20 & 0.545 & 3.311 & 2046.444 & \\
\hline & 10 & 10 & 1.165 . & 3.361 & 2296.14 & \\
\hline & 25 & 4 & 1.986 & 3.543 & 3491.403 & \\
\hline & 50 & 2 & 2.606 & 3.767 & $\mathbf{5 8 4 7 . 9 0 0}$ & \\
\hline & 100 & 1 & 3.226 & 3.818 & 6576.578 & \\
\hline & 200 & 0.5 & 3.936 & 4.061 & 11508.003 & \\
\hline
\end{tabular}

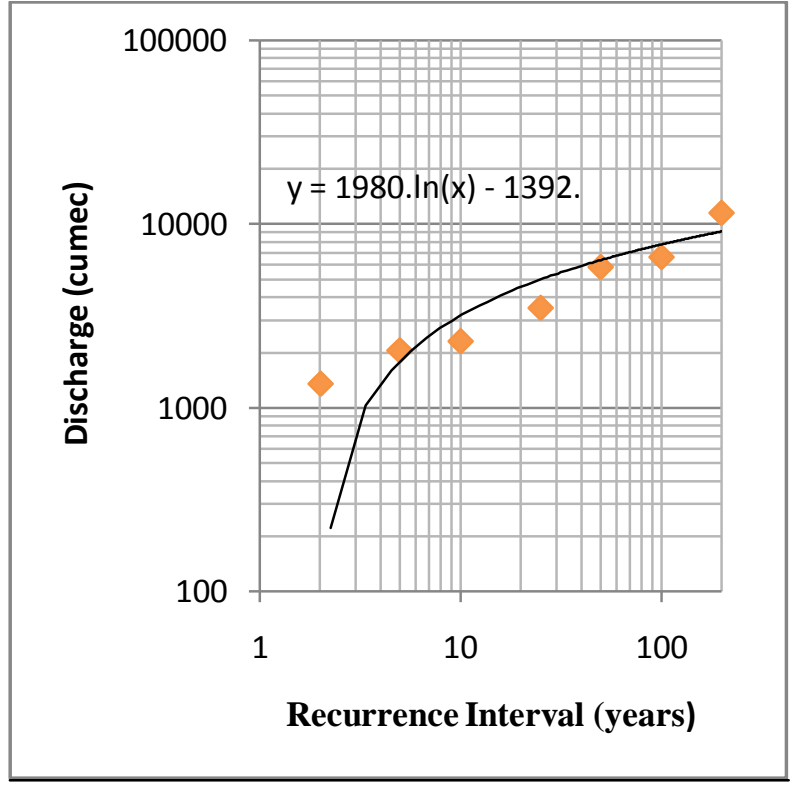

Figure 1: Relation between discharge and return period

Table 4 Calculation of Discharges for return periods for River Gauge Stations

\begin{tabular}{|c|c|c|c|c|c|c|}
\hline $\begin{array}{l}\text { Gauge } \\
\text { Staion }\end{array}$ & $\begin{array}{l}\text { Frequen } \\
\text { cy factor } \\
\text { K }\end{array}$ & $\begin{array}{l}\text { Return } \\
\text { period } \\
\text { T(yrs) }\end{array}$ & & $\begin{array}{l}y i=\log Q \\
\text { yi }=\bar{y}+ \\
X S y\end{array}$ & $\mathrm{X} i=\mathrm{Q} \mathrm{m} 3 / \mathrm{s}$ & $\begin{array}{l}\text { Relation between } \\
\text { Expected } \\
\text { Discharge and } \\
\text { Return Period }\end{array}$ \\
\hline \multirow{7}{*}{ Argunwad } & \multirow{7}{*}{$\begin{array}{l}\mathrm{g}= \\
0.2682\end{array}$} & 2 & -0.029 & 3.558 & 3610 & \multirow{7}{*}{$\begin{array}{l}y=1662 . \ln (x)+ \\
2430 .\end{array}$} \\
\hline & & 5 & 0.831 & 3.712 & 5149 & \\
\hline & & 10 & 1.299 & 3.796 & 6245.8 & \\
\hline & & 25 & 1.812 & 3.887 & 7715.3 & \\
\hline & & 50 & 2.148 & 3.948 & 8864.7 & \\
\hline & & 100 & 2.457 & 4.003 & 10069 & \\
\hline & & 200 & 2.744 & 4.054 & 11333 & \\
\hline \multirow{7}{*}{ Karad } & \multirow{7}{*}{$\begin{array}{l}g= \\
0.0112\end{array}$} & 2 & -0.001 & 3.413 & 2590.5 & \multirow{7}{*}{$\begin{array}{l}y=1615 . \ln (x)+ \\
1405\end{array}$} \\
\hline & & 5 & 0.842 & 3.606 & 4034.9 & \\
\hline & & 10 & 1.283 & 3.707 & 5088.6 & \\
\hline & & 25 & 1.754 & 3.814 & 6518.2 & \\
\hline & & 50 & 2.058 & 3.884 & 7650 & \\
\hline & & 100 & 2.332 & 3.946 & 8833.9 & \\
\hline & & 200 & 2.584 & 4004 & 10083 & \\
\hline
\end{tabular}


Flood Frequency Analysis of Upper Krishna River Basin catchment area using Log Pearson Type III

\begin{tabular}{|c|c|c|c|c|c|c|}
\hline \multirow{7}{*}{$\begin{array}{l}\text { Kurundwa } \\
\text { d }\end{array}$} & \multirow{7}{*}{$\begin{array}{l}g=- \\
0.0215\end{array}$} & 2 & 0.081 & 3.675 & 4731.2 & \multirow{7}{*}{$\begin{array}{l}y=981.4 \ln (x)+ \\
4431\end{array}$} \\
\hline & & 5 & 0.859 & 3.788 & 6139 & \\
\hline & & 10 & 1.221 & 3.841 & 6931.9 & \\
\hline & & 25 & 1.574 & 3.892 & 7804.1 & \\
\hline & & 50 & 1.788 & 3.923 & 8383.5 & \\
\hline & & 100 & 1.967 & 3.949 & 8901.3 & \\
\hline & & 200 & 2.120 & 3.972 & 9369.6 & \\
\hline \multirow{7}{*}{ Sadalgi } & \multirow{7}{*}{$\begin{array}{l}g=- \\
0.4028\end{array}$} & 2 & -0.048 & 3.295 & 1973.4 & \multirow{7}{*}{$\begin{array}{l}y=4020 . \ln (x)- \\
2395\end{array}$} \\
\hline & & 5 & 0.825 & 3.603 & 4004.8 & \\
\hline & & 10 & 1.311 & 3.774 & 5940.6 & \\
\hline & & 25 & 1.848 & 3.963 & 9186.9 & \\
\hline & & 50 & 2.208 & 4.090 & 12299 & \\
\hline & & 100 & 2.539 & 4.207 & 16096 & \\
\hline & & 200 & 2.851 & 4.316 & 20725 & \\
\hline \multirow{7}{*}{ Samdoli } & \multirow{7}{*}{$\begin{array}{l}\mathrm{g}= \\
1.7248\end{array}$} & 2 & -0.423 & 3.182 & 1521.2 & \multirow{7}{*}{$\begin{array}{l}y=5975 . \ln (x)- \\
7428\end{array}$} \\
\hline & & 5 & 0.472 & 3.402 & 2521.9 & \\
\hline & & 10 & 1.257 & 3.595 & 3931.6 & \\
\hline & & 25 & 2.334 & 3.859 & 7223.6 & \\
\hline & & 50 & 3.181 & 4.066 & 11654 & \\
\hline & & 100 & 4.054 & 4.281 & 19084 & \\
\hline & & 200 & 4.934 & 4.497 & 31375 & \\
\hline \multirow{7}{*}{ Terwad } & \multirow{7}{*}{$\begin{array}{l}g=- \\
0.3109\end{array}$} & 2 & 0.084 & 3.248 & 1770 & \multirow{7}{*}{$\begin{array}{l}y=426.1 \ln (x)+ \\
1631 .\end{array}$} \\
\hline & & 5 & 0.857 & 3.375 & 2369.6 & \\
\hline & & 10 & 1.215 & 3.433 & 2712.3 & \\
\hline & & 25 & 1.564 & 3.491 & 3094.3 & \\
\hline & & 50 & 1.771 & 3.524 & 3345.2 & \\
\hline & & 100 & 1.945 & 3.553 & 3571.7 & \\
\hline & & 200 & 2.096 & 3.578 & 3782.3 & \\
\hline
\end{tabular}

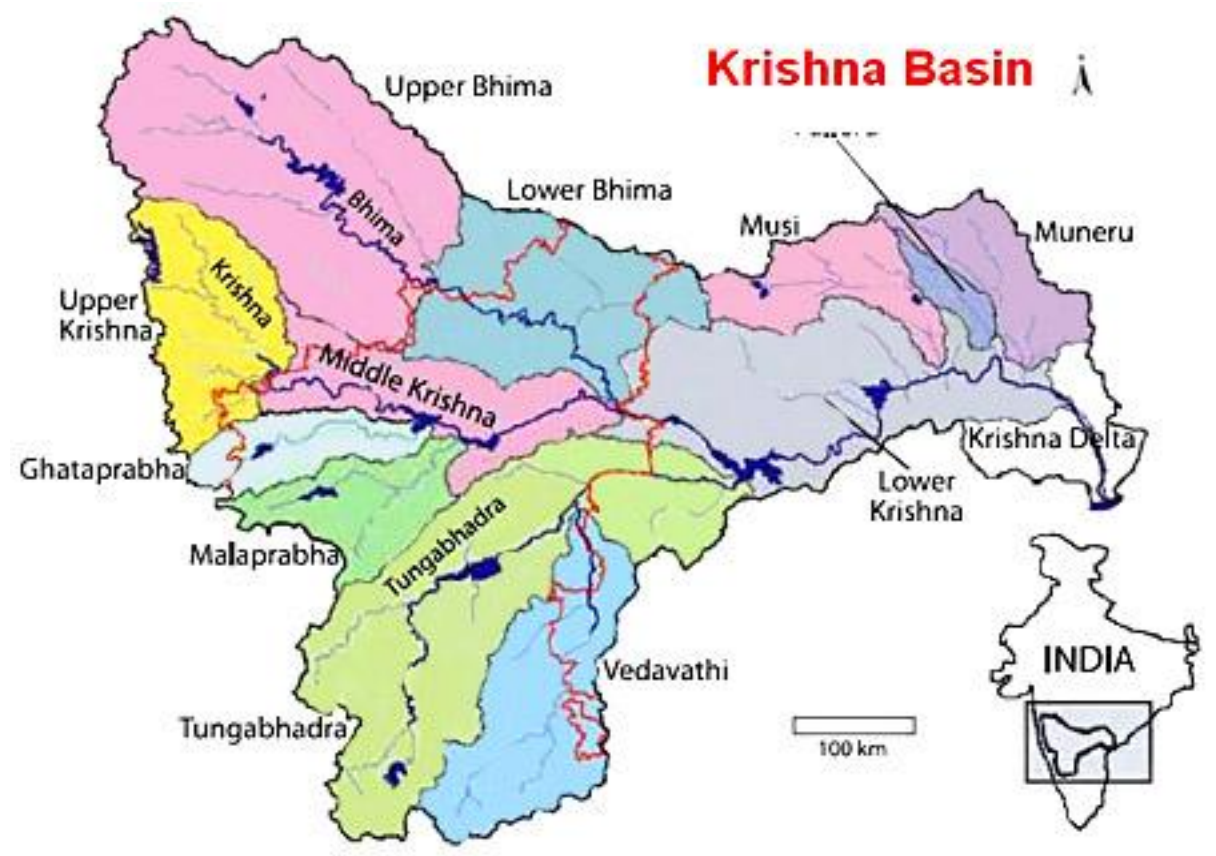




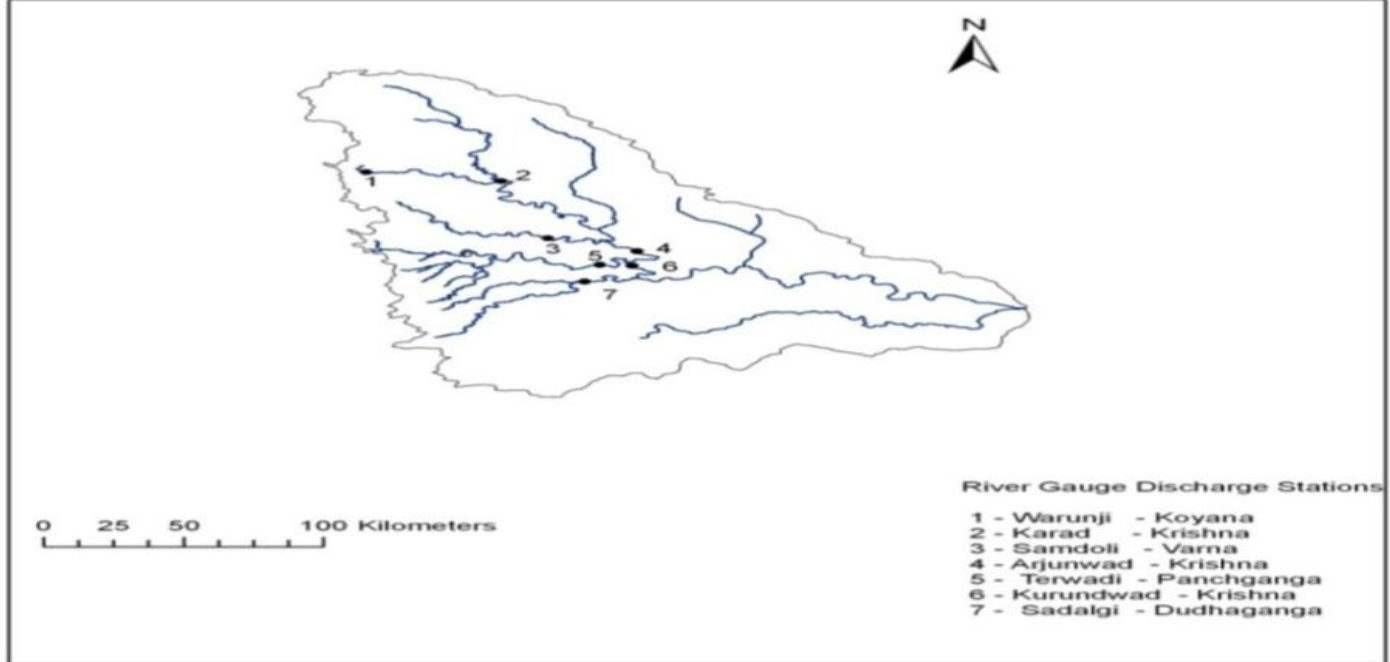

Figure 2: Study area

Table 5.Frequency Factors K for Gamma and log-Pearson Type III Distributions (Haan, 1977)

\begin{tabular}{|c|c|c|c|c|c|c|c|c|}
\hline & \multicolumn{8}{|c|}{ Recurrence Interval In Years } \\
\hline & 1.0101 & 2 & 5 & 10 & 25 & 50 & 100 & 200 \\
\hline SKEW COEFFICIENT & \multicolumn{8}{|c|}{ Percent Chance $(>=)=1-F$} \\
\hline Cs & 99 & 50 & 20 & 10 & 4 & 2 & 1 & 0.5 \\
\hline 3 & -0.667 & -0.396 & 0.420 & 1.180 & 2.278 & 3.152 & 4.051 & 4.970 \\
\hline 2.9 & -0.690 & -0.390 & 0.440 & 1.195 & 2.277 & 3.134 & 4.013 & 4.904 \\
\hline 2.8 & -0.714 & -0.384 & 0.460 & 1.210 & 2.275 & 3.114 & 3.973 & 4.847 \\
\hline 2.7 & -0.740 & -0.376 & 0.479 & 1.224 & 2.272 & 3.093 & 3.932 & 4.783 \\
\hline 2.6 & -0.769 & -0.368 & 0.499 & 1.238 & 2.267 & 3.071 & 3.889 & 4.718 \\
\hline 2.5 & -0.799 & -0.360 & 0.518 & 1.250 & 2.262 & 3.048 & 3.845 & 4.652 \\
\hline 2.4 & -0.832 & -0.351 & 0.537 & 1.262 & 2.256 & 3.023 & 3.800 & 4.584 \\
\hline 2.3 & -0.867 & -0.341 & 0.555 & 1.274 & 2.248 & 2.997 & 3.753 & 4.515 \\
\hline 2.2 & -0.905 & $-\mathbf{- 0 . 3 3 0}$ & 0.574 & 1.284 & 2.240 & 2.970 & 3.705 & 4.444 \\
\hline 2.1 & -0.946 & -0.319 & 0.592 & 1.294 & 2.230 & 2.942 & 3.656 & 4.372 \\
\hline 2 & -0.990 & -0.307 & 0.609 & 1.302 & 2.219 & 2.912 & 3.605 & 4.298 \\
\hline 1.9 & -1.037 & -0.294 & 0.627 & 1.310 & 2.207 & 2.881 & 3.553 & 4.223 \\
\hline 1.8 & -1.087 & -0.282 & 0.643 & 1.318 & 2.193 & 2.848 & 3.499 & 4.147 \\
\hline 1.7 & -1.140 & -0.268 & 0.660 & 1.324 & 2.179 & 2.815 & 3.444 & 4.069 \\
\hline 1.6 & -1.197 & -0.254 & 0.675 & 1.329 & 2.163 & 2.780 & 3.388 & 3.990 \\
\hline 1.5 & -1.256 & -0.240 & 0.690 & 1.333 & 2.146 & 2.743 & 3.330 & 3.910 \\
\hline 1.4 & -1.318 & -0.225 & 0.705 & 1.337 & 2.128 & 2.706 & 3.271 & 3.828 \\
\hline 1.3 & -1.383 & -0.210 & 0.719 & 1.339 & 2.108 & 2.666 & 3.211 & 3.745 \\
\hline 1.2 & -1.449 & -0.195 & 0.732 & 1.340 & 2.087 & 2.626 & 3.149 & 3.661 \\
\hline 1.1 & -1.518 & -0.180 & 0.745 & 1.341 & 2.066 & 2.585 & 3.087 & 3.575 \\
\hline 1 & -1.588 & -0.164 & 0.758 & 1.340 & 2.043 & 2.542 & 3.022 & 3.489 \\
\hline 0.9 & -1.660 & -0.148 & 0.769 & 1.339 & 2.018 & 2.498 & 2.957 & 3.401 \\
\hline
\end{tabular}


Flood Frequency Analysis of Upper Krishna River Basin catchment area using Log Pearson Type III

\begin{tabular}{|c|c|c|c|c|c|c|c|c|}
\hline 0.8 & -1.733 & -0.132 & 0.780 & 1.336 & 1.993 & 2.453 & 2.891 & 3.312 \\
\hline 0.7 & -1.806 & -0.116 & 0.790 & 1.333 & 1.967 & 2.407 & 2.824 & 3.223 \\
\hline 0.6 & -1.880 & -0.099 & 0.800 & 1.328 & 1.939 & 2.359 & 2.755 & 3.132 \\
\hline 0.5 & -1.955 & -0.083 & 0.808 & 1.323 & 1.910 & 2.311 & 2.686 & 3.041 \\
\hline 0.4 & -2.029 & -0.066 & 0.816 & 1.317 & 1.880 & 2.261 & 2.615 & 2.949 \\
\hline 0.3 & -2.104 & -0.050 & 0.824 & 1.309 & 1.849 & 2.211 & 2.544 & 2.856 \\
\hline 0.2 & -2.178 & -0.033 & 0.830 & 1.301 & 1.818 & 2.159 & 2.472 & 2.763 \\
\hline 0.1 & -2.252 & -0.017 & 0.836 & 1.292 & 1.785 & 2.107 & 2.400 & 2.67 \\
\hline o & -2.326 & 0.000 & 0.842 & 1.282 & 1.751 & 2.054 & 2.326 & 2.576 \\
\hline-0.1 & -2.4 & 0.017 & 0.846 & 1.27 & 1.716 & 2.000 & 2.252 & 2.482 \\
\hline-0.2 & -2.472 & $\mathbf{0 . 0 3 3}$ & 0.850 & 1.258 & 1.680 & 1.945 & 2.178 & 2.388 \\
\hline-0.3 & -2.544 & 0.050 & 0.853 & 1.245 & 1.643 & 1.890 & 2.104 & 2.294 \\
\hline-0.4 & -2.615 & 0.066 & 0.855 & 1.231 & 1.606 & 1.834 & 2.029 & 2.201 \\
\hline-0.5 & -2.686 & 0.083 & 0.856 & 1.216 & 1.567 & 1.777 & 1.955 & 2.108 \\
\hline-0.6 & -2.755 & 0.099 & 0.857 & 1.200 & 1.528 & 1.720 & 1.880 & 2.016 \\
\hline-0.7 & -2.824 & 0.116 & 0.857 & 1.183 & 1.488 & 1.663 & 1.806 & 1.926 \\
\hline-0.8 & -2.891 & 0.132 & 0.856 & 1.166 & 1.448 & 1.606 & 1.733 & 1.837 \\
\hline-0.9 & -2.957 & 0.148 & 0.854 & 1.147 & 1.407 & 1.549 & 1.660 & 1.749 \\
\hline-1 & -3.022 & 0.164 & 0.852 & 1.128 & 1.366 & 1.492 & 1.588 & 1.664 \\
\hline-1.1 & -3.087 & 0.180 & 0.848 & 1.107 & 1.324 & 1.435 & 1.518 & 1.581 \\
\hline-1.2 & -3.149 & 0.195 & 0.844 & 1.086 & 1.282 & 1.379 & 1.449 & 1.501 \\
\hline-1.3 & -3.211 & 0.210 & 0.838 & 1.064 & 1.240 & 1.324 & 1.383 & 1.424 \\
\hline-1.4 & -3.271 & 0.225 & 0.832 & 1.041 & 1.198 & 1.270 & 1.318 & 1.351 \\
\hline-1.5 & -3.33 & 0.240 & 0.825 & 1.018 & 1.157 & 1.217 & 1.256 & 1.282 \\
\hline-1.6 & -3.880 & 0.254 & 0.817 & 0.994 & 1.116 & 1.166 & 1.197 & 1.216 \\
\hline-1.7 & -3.444 & 0.268 & 0.808 & 0.970 & 1.075 & 1.116 & 1.140 & 1.155 \\
\hline-1.8 & -3.499 & 0.282 & 0.799 & 0.945 & 1.035 & 1.069 & 1.087 & 1.097 \\
\hline-1.9 & -3.553 & 0.294 & 0.788 & 0.920 & 0.996 & 1.023 & 1.037 & 1.044 \\
\hline-2 & -3.605 & 0.307 & 0.777 & 0.895 & 0.959 & 0.980 & 0.990 & 0.995 \\
\hline-2.1 & -3.656 & 0.319 & 0.765 & 0.869 & 0.923 & 0.939 & 0.946 & 0.949 \\
\hline-2.2 & -3.705 & 0.330 & 0.752 & 0.844 & 0.888 & 0.900 & 0.905 & 0.907 \\
\hline-2.3 & -3.753 & 0.341 & 0.739 & 0.819 & 0.855 & 0.864 & 0.867 & 0.869 \\
\hline-2.4 & -3.800 & 0.351 & 0.725 & 0.795 & 0.823 & 0.830 & 0.832 & 0.833 \\
\hline-2.5 & -3.845 & 0.360 & 0.711 & 0.711 & 0.793 & 0.798 & 0.799 & 0.800 \\
\hline-2.6 & -3.899 & 0.368 & 0.696 & 0.747 & 0.764 & 0.768 & 0.769 & 0.769 \\
\hline-2.7 & -3.932 & 0.376 & 0.681 & 0.724 & 0.738 & 0.740 & 0.740 & 0.741 \\
\hline-2.8 & -3.973 & 0.384 & 0.666 & 0.702 & 0.712 & 0.714 & 0.714 & 0.714 \\
\hline-2.9 & -4.013 & 0.390 & 0.651 & 0.681 & 0.683 & 0.689 & 0.690 & 0.690 \\
\hline-3 & -4.051 & 0.396 & 0.636 & 0.660 & 0.666 & 0.666 & 0.667 & 0.667 \\
\hline
\end{tabular}




\section{CONCLUSIONS}

Flood frequency analysis is one of the most challenging problems in hydrology. The hydrologic phenomena are often characterized by great variability and uncertainty precipitation, discharge. For this reason, a systematic approach to handling the problem is absolutely essential.

From the flood frequency study carried out on Upper Krishna River basin catchment for 2 yrs, 5yrs, 10yrs, 25yrs, 50yrs, 100yrs and $200 \mathrm{yrs}$ The estimated discharges obtained . It has been observed that design floods for return period of 2 year were flood to be almost same as the observed data and verified with historical data. Arjunwad river gauging station is having very high design flood as compare to other gauging station in the study area. These flood frequencies and design can be used as a guide in determining the capacity and design of structure like bridges, culverts.

\section{ACKNOWLEDGEMENT}

The author is pleased to acknowledge to Chief Er.C.A.Birajdhar and H.T.Dhumal, Department of Water recourse, Govt .of Maharashtra for availability of data and encouragement to write this paper.Greatful acknowledgement are also due to Prof. Nayan Sharma, Professor \&Head Department of WRD\&M, and Prof. Pradeep Kumar Garg, Indian Institute of Technology,Roorkee who reviewed and given insightful comments on drafting of this paper.

\section{REFERENCES}

[1] Akintola, J.O. 1986 Rainfall distribution in Nigeria 1892-1983, Impact publishers Nig. Ltd., Ibadan.

[2] Arora, K.R. 2007. Irrigation, Water Power and Water resources Engineering. Standard Publishers Distributors, New Delhi

[3] Asawa, G.L. 2005. Irrigation and Water resources Engineering. New Age International Ltd. Publishers,New Delhi

[4] Bhakar, S.R., Bansal, A.N. Chhajed, N., and Purohit, R.C. 2006. Frequency analysis of consecutive days maximum rainfall at Banswara, Rajasthan, India. ARPN Journal of Engineering and Applied Sciences. 1(3): 64-67.

[5] Borbda and Benin Owena 2005.River basin Hydrological year Book, 1995-1998

[6] Bruce, J.P., and Clark, P.H. 1988. Introduction to hydrometeorology. Pergamon Press, Oxford

[7] Chow, V.T., D.R.Maidment and L.W.Mays 1988. Applied Hydrology. McGraw Hill Book Company,Singapore.

[8] Garg P.K., K.P.Sharma.and S.C.Jain.1986 Satellite Remote Sensing for Mapping of Flood-Plains and Allied Features, Int. Symp. on Flood Frequency and Risk Analysis, Louisiana, USA.

[9] Haktan Y.T 1992. Comparison of Various flood frequency distribution using annual flood peaks data of rivers in Anatolis. Journal of Hydrology. Vol.136; pp1-31

[10] Haan, C.T. 1977. Statistical methods in hydrology. Ames, IA: Iowa State University Press.

[11] Ibrahim M.H., and E.A. Isiguzo, 2009. Flood Frequency Analysis of Guara River Catchment at Jere, Kaduna State, Nigeria, Scientific Research and Essay Vol. 4 (6), pp. 636 - 646.

[12] Jagadesh, T.R. and M.A.Jayaram 2009. Design of bridge Structures. 2nd Edition. PHI Learning PVT Ltd, New Delhi India

[13] Prasuhn, A. 1992. Fundamentals of Hydraulic Engineering. Oxford University Press New York

[14] O.C.Izinyon,N.Ihimkpen. Flood Frequency Analysis of Ikpoba River Catchment at Benin city Using Log Prearson Type III

[15] Distribution, Journal of Emerging Trends in Engineering and Applied Sciences Vol.2(1) pp-50-55

[16] Ojha, G.S.P.,R Berndtsson .and P.Bhunya 2008. Engineering Hydrology. 1st Edition, Oxford University Press. New Delhi, India

[17] Sankhua, R.N.N. Sharma, P.K.Garg, and A.D.Pandey, 2005. Use of remote sensing and ANN in assessment of erosion activities in Majuli, the world's largest river island: Int. Journal of Remote Sensing, Vol. 26, pp. 4445 - 4454.

[18] Upadhaya, A., and Singh, S.R. 1998. Estimation of consecutive days Maximum Rainfall by Various Methods and their Comparison. Indian Journal of S. Cons. 26(2): 193-2001. 